\title{
Magnetic field driven transition from an antiferromagnetic ground state to a ferrimagnetic state in $\mathrm{Rb}_{0.19} \mathrm{Ba}_{0.3} \mathrm{Mn}_{1.1}\left[\mathrm{Fe}(\mathrm{CN})_{6}\right] \cdot 0.48 \mathrm{H}_{2} \mathrm{O}$ Prussian blue analogue
}

\author{
S. M. Yusuf, ${ }^{1, a)}$ N. Thakur, ${ }^{1}$ M. Medarde, ${ }^{2}$ and L. Keller ${ }^{3}$ \\ ${ }^{1}$ Solid State Physics Division, Bhabha Atomic Research Centre, Mumbai 400085 , India \\ ${ }^{2}$ Laboratory for Developments and Methods, Paul Scherrer Institut, CH-5232 Villigen PSI, Switzerland \\ ${ }^{3}$ Laboratory for Neutron Scattering, Paul Scherrer Institut, CH-5232 Villigen PSI, Switzerland
}

(Received 12 June 2012; accepted 25 September 2012; published online 1 November 2012)

\begin{abstract}
We report a magnetic field dependent $(H)$ antiferromagnetic (AFM) to ferrimagnetic (FIM) transition in $\mathrm{Rb}_{0.19} \mathrm{Ba}_{0.3} \mathrm{Mn}_{1.1}\left[\mathrm{Fe}(\mathrm{CN})_{6}\right] \cdot 0.48 \mathrm{H}_{2} \mathrm{O}$ Prussian blue analogue. A microscopic understanding of the nature of this field induced AFM to FIM transition is achieved by carrying out a low temperature neutron diffraction study under an external magnetic field. A decrease in Néel temperature and an evolution of a ferrimagnetic phase with an increase in dc magnetic field are evident in dc magnetic field dependent ac susceptibility measurements. With an increase in $H$, the FIM phase grows at the expense of the AFM phase, and for $H>4 \mathrm{~T}$, the system fully transforms to the FIM phase. A magnetic structure consisting of an antiparallel stacking of ferrimagnetic sheets along crystallographic $c$-direction is proposed for the FIM phase. Based on the results obtained, a phase diagram describing the evolution of both magnetic phases under external magnetic field is presented. (C) 2012 American Institute of Physics. [http://dx.doi.org/10.1063/1.4759361]
\end{abstract}

\section{INTRODUCTION}

The current trend in the evergreen field of molecular magnetism ${ }^{1-4}$ is to investigate multifunctional materials. A molecular bistability is exhibited by multifunctional molecular materials and it is possible to tune their magnetic properties by changing an external parameter. The external parameter could be light, pressure, humidity, etc. These materials can be potentially used in a number of practical applications, e.g., molecular electronics, information storage, etc. In this regard, the Prussian blue analogue (PBA) family ${ }^{5-27}$ constitutes a very popular class of candidate materials for multifunctional use. Upon application of an external stimulus, a number of PBAs show a switching between two electronic states such as photo-induced magnetization, ${ }^{23}$ pressure induced magnetization, ${ }^{28}$ humidity induced magnetization, ${ }^{21}$ and spin crossover. ${ }^{29}$ Magnetic field may also act as an external stimulus to induce two different magnetic states in a material. For example, some antiferromagnets can display a phase transition to another magnetic state (with a net magnetic moment) on application of an external magnetic field. ${ }^{30}$ This phenomenon is possible due to the presence of competing magnetic exchange interactions in the material. Such kind of magnetic field-induced phase transitions have been investigated in a number of different materials. ${ }^{30}$ Some prominent examples of this kind of materials are $\mathrm{FeCl}_{2},{ }^{31-35}\left[\left(\mathrm{CH}_{3}\right)_{3} \mathrm{NH}\right]$ $\mathrm{CoCl}_{3} \cdot 2 \mathrm{H}_{2} \mathrm{O},{ }^{36} \mathrm{CoCl}_{2} \cdot 2 \mathrm{H}_{2} 0,{ }^{35,37}$ etc. In the $\mathrm{CN}$-bridged molecular magnets, such kind of magnetic field dependent phase transitions have been also reported. Good examples are $\left\{\left[(\mathrm{Tp}) \mathrm{Fe}^{3+}(\mathrm{CN})_{3}\right]_{2} \mathrm{Mn}^{2+}(\mathrm{DMF})_{2}\right\}_{\mathrm{n}},{ }^{38} \quad[\mathrm{Ni}(\text { cyclam })]_{3}$

\footnotetext{
a) Author to whom correspondence should be addressed. Electronic mail: smyusuf@barc.gov.in. Fax: +91 22 25505151. Telephone: +91 22 25595608
}

$\left[\mathrm{Fe}(\mathrm{CN})_{6}\right]_{2} \cdot n \mathrm{H}_{2} \mathrm{O},{ }^{39} \quad\left[\mathrm{~K}(18-\mathrm{cr})(2 \mathrm{PrOH})_{2}\right] \quad\left[\{\mathrm{Mn}(\text { acacen })\}_{2}\right.$ $\left.\left\{\mathrm{Fe}(\mathrm{CN})_{6}\right\}\right],{ }^{40}\left\{\left[\mathrm{Ni}(\mathrm{en})_{2}\right]_{3}\left[\mathrm{Fe}(\mathrm{CN})_{6}\right]_{2} \cdot x \mathrm{H}_{2} \mathrm{O}\right\}_{n},{ }^{41} \mathrm{~K}_{0.2}\left(\mathrm{Mn}^{2+}\right)_{0.66}$ $\left(\mathrm{Mn}^{3+}\right)_{1.44}\left[\left(\mathrm{Fe}^{2+}\right)_{0.2}\left(\mathrm{Fe}^{3+}\right)_{0.8}(\mathrm{CN})_{6}\right] \mathrm{O}_{0.66}\left(\mathrm{CH}_{3} \mathrm{COO}\right)_{1.32} \cdot 7.6 \mathrm{H}_{2} \mathrm{O},{ }^{42}$ etc. The magnetic structures of these compounds can be described as AF-coupled stacks of ferrimagnetic/ferromagnetic layers, and phase transitions occur due to a competition between the intra and inter-layer magnetic exchange interactions. ${ }^{43}$ On applying an external magnetic field in the antiferromagnetic state, the compounds transform to fieldinduced ferri/ferromagnetic state. ${ }^{43}$

In our earlier work on $\mathrm{Rb}_{x} \mathrm{Ba}_{y} \mathrm{Mn}_{[3-(x+2 y)] / 2}[\mathrm{Fe}$ $\left.(\mathrm{CN})_{6}\right] \cdot z \mathrm{H}_{2} \mathrm{O}$ PBAs, ${ }^{44}$ we proposed a possible switching between two magnetic states (antiferromagnetic to ferrimagnetic) upon application of magnetic field. In that work, ${ }^{44} \mathrm{dc}$ magnetization and zero field neutron diffraction measurements were employed. At $1.5 \mathrm{~K}$ under zero magnetic field, the analysis of the neutron powder diffraction data revealed a 3D long-range antiferromagnetic ordering in these compounds. The magnetic structure is layered, consisting of an antiparallel stacking of ferromagnetic sheets along crystallographic $c$-direction. However, dc magnetization study indicated that an antiferromagnetic to ferrimagnetic-like phase transition occurred on application of an external magnetic field. The variations of $\mathrm{Mn}^{2+}$ and $\mathrm{Fe}^{3+}$ ordered magnetic moments as a function of temperature as well as the layered antiferromagnetic structure of the compounds were also described in the earlier work. ${ }^{44}$ The experimental studies on other $\mathrm{CN}$ based molecular magnets (such as $\left[\mathrm{Ni}(\text { cyclam) }]_{3}\right.$ $\left[\mathrm{Fe}(\mathrm{CN})_{6}\right]_{2} \cdot n \mathrm{H}_{2} \mathrm{O}^{39}$ and $\left.\left\{\left[\mathrm{Ni}(\mathrm{en})_{2}\right]_{3}\left[\mathrm{Fe}(\mathrm{CN})_{6}\right]_{2} \cdot x \mathrm{H}_{2} \mathrm{O}\right\}_{n}{ }^{41}\right)$, which show field induced magnetic phase transition, are based on bulk techniques such as magnetization and resistivity. However, there is no microscopic understanding of the nature of antiferromagnetic to ferrimagnetic/ferromagneticlike phase transitions. In fact, this motivated us to investigate 
the field-induced magnetic phase transition in $\mathrm{Rb}_{0.19} \mathrm{Ba}_{0.3}$ $\mathrm{Mn}_{1.1}\left[\mathrm{Fe}(\mathrm{CN})_{6}\right] \cdot 0.48 \mathrm{H}_{2} \mathrm{O}$ PBA with the help of magnetic field dependent neutron diffraction. Besides, field dependent ac susceptibility measurements have been carried out in the present study. A quantitative analysis for both antiferromagnetic and field induced ferrimagnetic phases including a magnetic phase diagram for the compound is given in the present study. Such tunable PBAs which undergo controlled changes of their molecular states in response to external perturbations may find applications in molecular electronics such as bi-stable memory devices.

\section{EXPERIMENTAL SECTION}

Polycrystalline $\quad \mathrm{Rb}_{0.19} \mathrm{Ba}_{0.3} \mathrm{Mn}_{1.1}\left[\mathrm{Fe}(\mathrm{CN})_{6}\right] \cdot 0.48 \mathrm{H}_{2} \mathrm{O}$ compound was synthesized by a co-precipitation method, as described in our earlier work. ${ }^{44}$ Neutron diffraction study was carried out under applied magnetic field (range: 0-2.7 T) over $1-5-20 \mathrm{~K}$ using the DMC powder diffractometer $(\lambda=$ $2.4585 \AA$ A), SINQ, Paul Scherrer Institute, Switzerland. To avoid the displacement of the crystallites within the sample holder under the action of the field, a compressed rod was used. The diffraction data were analyzed by the Rietveld method using the FULLPROF program. ${ }^{45}$ The real part of the ac susceptibility at a frequency of $133 \mathrm{~Hz}$ under different values of superimposed dc magnetic field has been measured using a Quantum Design Physical Properties Measuring System device (PPMS). For each iso-field measurement, the sample was first cooled from 40 to $2 \mathrm{~K}$ under dc field, and the ac susceptibility measurements have been then carried out (keeping the dc field on) in the warming cycle.

\section{RESULTS AND DISCUSSION}

Figures 1 and 2 show the observed and Rietveld refined neutron diffraction patterns for the compound $\mathrm{Rb}_{0.19} \mathrm{Ba}_{0.3} \mathrm{Mn}_{1.1}\left[\mathrm{Fe}(\mathrm{CN})_{6}\right] \cdot 0.48 \mathrm{H}_{2} \mathrm{O}$ at $1.5 \mathrm{~K}$ under applied magnetic field $H$ (range: $0-2.7 \mathrm{~T}$ ). In our earlier work, ${ }^{44}$ we already discussed that at zero magnetic field, the appearance of extra Bragg peaks at scattering vector magnitudes, $Q \sim$ $0.84 \AA^{-1}, 1.33 \AA^{-1}$, and $1.79 \AA^{-1}$ [as shown in Fig. 1(a)] confirmed a $3 \mathrm{D}$ long-range antiferromagnetic ordering for the present sample. The extra (magnetic) Bragg peaks were indexed as (100), (111), and (201) with respect to the chemical unit cell. The intensities of these AFM peaks decrease with increasing $H$. On the other hand, the increment in $H$ leads to an increase in intensity of fundamental Bragg peaks (101), (200) and (211). This increase in intensity may be ascribed to the development of a ferromagnetic (FM) or FIM structure of the compound. Our detailed study has shown that the state is a FIM (discussed later). The Rietveld refinement of neutron diffraction data at $1.5 \mathrm{~K}$ under zero magnetic field was carried out by considering both nuclear and antiferromagnetic phases, and the size of the magnetic unit cell was found to be same as the chemical unit cell, as reported in our earlier work. ${ }^{44}$ With the help of Rietveld refinement of neutron diffraction data, the water content in the sample was estimated to be 0.48 per formula unit. The Rietveld refinement of neutron diffraction data at $1.5 \mathrm{~K}$ under applied field $0.5 \leq H \leq 2.7 \mathrm{~T}$ has been carried out by considering the

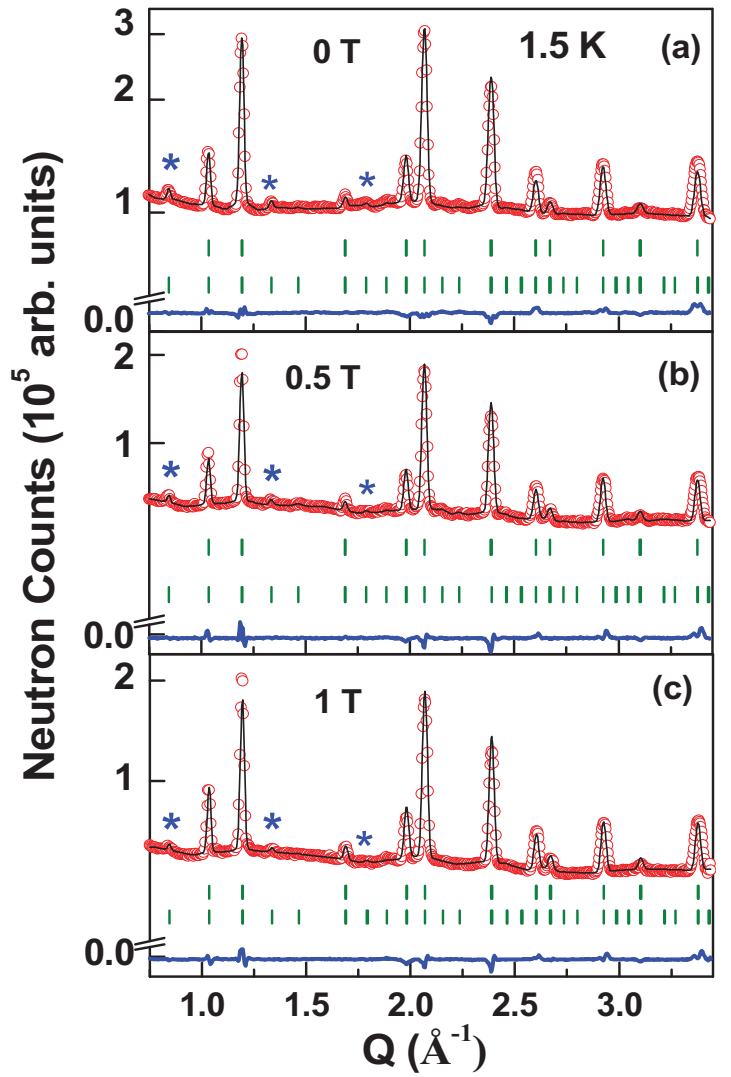

FIG. 1. Rietveld refined neutron-diffraction patterns for $\mathrm{Rb}_{0.19} \mathrm{Ba}_{0.3} \mathrm{Mn}_{1.1}$ $\left[\mathrm{Fe}(\mathrm{CN})_{6}\right] \cdot 0.48 \mathrm{H}_{2} \mathrm{O}$ compound at $1.5 \mathrm{~K}$ under different applied magnetic fields $(0-1 \mathrm{~T})$. The observed and calculated diffraction patterns are indicated by circles and solid line, respectively. Solid line at the bottom shows the difference between observed and calculated patterns. In (a), the vertical bars indicate the allowed Bragg peaks position for nuclear (top row) and antiferromagnetic (bottom row) phase. In (b) and (c), the vertical bars indicate the allowed Bragg peaks position for nuclear and ferrimagnetic (top row) and antiferromagnetic (bottom row) phases. Asterisks mark the AFM Bragg peaks. Here the observed and calculated diffraction patterns are plotted in a semi-log scale, while the difference is plotted in a linear scale.

nuclear, and the antiferromagnetic phases along with a new ferrimagnetic phase. The important structural parameters (atomic coordinates, and lattice parameters), derived from the analysis, are given in Table I. Here, different models have been tried out. The first is the inhomogeneous model, in which both AFM and FIM phases coexist. As we change the magnetic field value, the volume fraction of the two phases changes. The second one is the homogeneous model, where the AFM and FIM phases result from a canted magnetic structure. Other possible models including the one with a coexistence of AFM and FM magnetic phases are also considered. We found that the inhomogeneous model with phase coexistence is the only one to provide satisfactory fits of the data. In the pure AFM model under zero magnetic field (discussed in our previous work ${ }^{44}$ ), the magnetic structure is layered, consisting of an antiparallel stacking of ferromagnetic sheets along crystallographic $c$-direction. Within each sheet in $a b$ plane, the ordered moments of $\mathrm{Mn}^{2+}$ and $\mathrm{Fe}^{3+}$ are aligned ferromagnetically along $c$-axis. If the magnetic moments of $\mathrm{Mn}^{2+}$ and $\mathrm{Fe}^{3+}$ are canted at a fixed angle to the unit cell edge, then the resolved components would give both AFM and FIM contributions. In this uniformly canted 


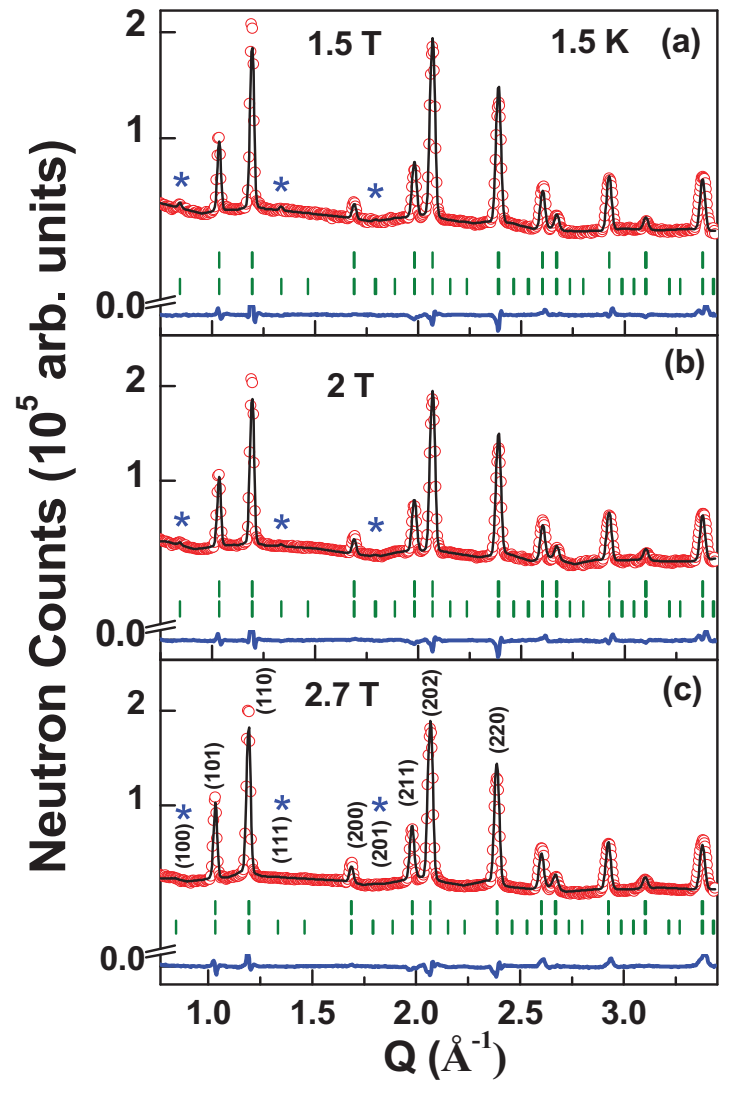

FIG. 2. Rietveld refined neutron-diffraction patterns at $1.5 \mathrm{~K}$ under different applied magnetic fields $(1.5-2.7 \mathrm{~T})$. The observed and calculated diffraction patterns are indicated by circles and solid line, respectively. Solid line at the bottom shows the difference between observed and calculated patterns. The vertical bars indicate the allowed Bragg peaks position for nuclear and ferrimagnetic (top row) and antiferromagnetic (bottom row) phases. Asterisks mark the AFM Bragg peaks. The observed and calculated diffraction patterns are plotted in a semi-log scale, while the difference is plotted in a linear scale.

magnetic structure, the vertical component of the moment (along the crystallographic $c$-axis) would give the AFM contribution, and the horizontal component (in the $a b$ plane) should give the FIM contribution. Therefore, the AFM and FIM moments should be perpendicular to each other. But in the present case, the intensity of the antiferromagnetic peaks and ferrimagnetic (fundamental) Bragg peaks fits only if we consider the moment direction to be along the crystallographic $c$-axis. So our neutron diffraction data infer the coexistence of both AFM and FIM phases (for $0 \leq H \leq 2.7 \mathrm{~T}$ ) under the inhomogeneous model. It may be noted that the refinement of the field dependent neutron diffraction data on considering a mixture of antiferromagnetic and ferromagnetic magnetic phases does not provide a good fit.

The variations of integrated intensities of the AFM Bragg peaks [(100) and (111)] as a function of $H$ at $1.5 \mathrm{~K}$ and $8 \mathrm{~K}$ are shown in Figs. 3(a) and 3(b), respectively. It is observed that the intensities of the AFM peaks decrease on increasing $H$ at both temperatures. It may also be noted that at $1.5 \mathrm{~K}$, a very small contribution to the AFM peaks exists even at $2.7 \mathrm{~T}$. However, at $8 \mathrm{~K}$, the AFM contribution totally vanishes under $2.7 \mathrm{~T}$. At $12 \mathrm{~K}$, no AFM peaks are observed for any value of $H$ (Fig. 3(c)). The arrows in Fig. 3(c) mark the expected positions of the (100) and (111) AFM peaks.
The variations of the integrated intensities of the fundamental Bragg peaks [(101), (200), and (211)] as a function of applied magnetic field at $1.5,8$, and $12 \mathrm{~K}$ are shown in Figs. 4(a)-4(c), respectively. An increase in intensity (without any broadening of the peak profiles as evident from Figs. 1, 2, and 3(c)), on increasing applied magnetic fields, gives a direct evidence of the development of a long-range ferrimagnetic order at higher fields at the temperatures $1.5,8$, and $12 \mathrm{~K}$. At zero field, the intensities of fundamental (101), (110), (200), (211) and (202) Bragg peaks do not show any enhancement with decrease in temperature down to $1.5 \mathrm{~K}$ (Fig. 4(d)), thereby asserting that the sample does not contain any ferrimagnetic phase at zero applied magnetic field. Here, a significant observation in Fig. 3(c) is that the sample is paramagnetic at $12 \mathrm{~K}$ under zero field. However, the enhancement of intensities of fundamental Bragg peaks [(101), (200) and (211)] at $12 \mathrm{~K}$ with an increasing $H$ confirms the presence of the ferrimagnetic phase even at $12 \mathrm{~K}$ at higher fields (Fig. 4(c)). At a further higher temperature $(20 \mathrm{~K})$, no enhancement in the intensities of fundamental Bragg peaks [(101), (200) and (211)] as a function of applied magnetic field is observed [Fig. 4(e)] indicating a pure paramagnetic (PM) character of the compound (at $20 \mathrm{~K}$ ). Figure 5 shows the variation of the AFM and FIM phase fractions with the $H$ at low temperature. It is observed that: (i) at both $1.5 \mathrm{~K}$ and $8 \mathrm{~K}$, the compound is wholly antiferromagnetic under zero field; (ii) at $1.5 \mathrm{~K}$, the AFM and FIM phases coexist for $H \geq 0.5 \mathrm{~T}$. However, at $8 \mathrm{~K}$, the AFM and FIM phases coexist for $0.5 \leq H<2.7 \mathrm{~T}$; (iii) at $8 \mathrm{~K}$, the AFM phase vanishes totally at $2.7 \mathrm{~T}$ and the compound is wholly ferrimagnetic.

Under zero external magnetic field, the compound has a layered antiferromagnetic structure with $T_{\mathrm{N}} \sim 11 \mathrm{~K}$ in which the magnetic coupling between layers in the $a b$-plane (e.g., between layers 1 and 2) is antiferromagnetic [Fig. 6(a)]. ${ }^{44}$ Within a layer, $\mathrm{Mn}(S=5 / 2)$ and $\mathrm{Fe}(S=1 / 2)$ spins are aligned in parallel direction along crystallographic $c$-axis whereas, the ferrimagnetic structure of the compound at $1.5 \mathrm{~K}$ under magnetic field consists of antiparallel alignment of $\mathrm{Mn}^{2+}$ and $\mathrm{Fe}^{3+}$ moments in the $a b$-plane, aligned along crystallographic c-axis as shown in Fig. 6(b). Thus, there is a net ferrimagnetic moment in the $a b$ plane and these ferrimagnetic sheets are stacked in antiparallel directions along the crystallographic $c$-axis, as shown in Fig. 6(b). Thus, under field, $\mathrm{Mn}^{2+}$ and $\mathrm{Fe}^{3+}$ spins are aligned in opposite directions both within the $a b$ layers and along the $c$ axis giving the compounds a ferrimagnetic ordering. In Fig. 6, a schematic illustration of the coupling of magnetic orbitals of $\mathrm{Mn}^{2+}$ and $\mathrm{Fe}^{3+}$ ions via $\mathrm{CN}$ orbitals along $z(c)$ axis as well as in the $x y(a b)$ plane is also shown to understand the magnetic exchange interactions. A detailed discussion of this point will be presented later in the text.

In order to investigate the variation of antiferromagnetic $\left(T_{\mathrm{N}}\right)$ as well as ferrimagnetic $\left(T_{\mathrm{C}}\right)$ transition temperatures with $H$, we have carried out ac susceptibility measurements under dc magnetic field. Figure 7(a) shows the temperature dependence of the real part of the ac susceptibility $\left(\chi_{\mathrm{ac}}\right)$ under different applied dc magnetic fields (range: 0-4 T). For $H=0$, a peak like feature is observed at $T_{\mathrm{N}} \sim 11 \mathrm{~K}$ in the $\chi_{\mathrm{ac}}$ 
TABLE I. Results of the Rietveld refinement for $\mathrm{Rb}_{0.19} \mathrm{Ba}_{0.3} \mathrm{Mn}_{1.1}\left[\mathrm{Fe}(\mathrm{CN})_{6}\right] \cdot 0.48 \mathrm{H}_{2} \mathrm{O}$ sample $\left(x^{\prime}, y^{\prime}\right.$ and $z^{\prime}$ denote the fractional coordinates) at $1.5 \mathrm{~K}$ at different values of $H$

\begin{tabular}{|c|c|c|c|c|c|c|}
\hline$H(\mathrm{~T})$ & Lattice parameters $(\AA)$ & Atom & Wyckoff site & $x^{\prime}$ & $y^{\prime}$ & $z^{\prime}$ \\
\hline \multirow[t]{9}{*}{2.7} & $\mathrm{a}=\mathrm{b}=7.436(4)$ & $\mathrm{Fe}$ & $2 a$ & 0 & 0 & 0 \\
\hline & $\mathrm{c}=10.53(1)$ & $\mathrm{Mn}$ & $2 b$ & 0 & 0 & 0.5 \\
\hline & & $\mathrm{Rb} 1$ & $2 \mathrm{c}$ & 0 & 0.5 & 0.25 \\
\hline & & $\mathrm{Rb} 2$ & $2 d$ & 0 & 0.5 & 0.75 \\
\hline & & Ba1 & $2 c$ & 0 & 0.5 & 0.25 \\
\hline & & $\mathrm{Ba} 2$ & $2 d$ & 0 & 0.5 & 0.75 \\
\hline & & $\mathrm{C}$ & $8 \mathrm{~g}$ & $0.204(2)$ & $0.204(2)$ & 0 \\
\hline & & $\mathrm{N}$ & $8 \mathrm{~g}$ & $0.280(6)$ & $0.280(6)$ & 0 \\
\hline & & $\mathrm{O}$ & $8 g$ & $0.280(6)$ & $0.280(6)$ & 0 \\
\hline \multirow[t]{9}{*}{2} & $\mathrm{a}=\mathrm{b}=7.436(5)$ & $\mathrm{Fe}$ & $2 a$ & 0 & 0 & 0 \\
\hline & $\mathrm{c}=10.53(2)$ & Mn & $2 b$ & 0 & 0 & 0.5 \\
\hline & & $\mathrm{Rb} 1$ & $2 c$ & 0 & 0.5 & 0.25 \\
\hline & & $\mathrm{Rb} 2$ & $2 d$ & 0 & 0.5 & 0.75 \\
\hline & & Ba1 & $2 \mathrm{c}$ & 0 & 0.5 & 0.25 \\
\hline & & $\mathrm{Ba} 2$ & $2 d$ & 0 & 0.5 & 0.75 \\
\hline & & $\mathrm{C}$ & $8 \mathrm{~g}$ & $0.204(9)$ & $0.204(9)$ & 0 \\
\hline & & $\mathrm{N}$ & $8 \mathrm{~g}$ & $0.280(4)$ & $0.280(4)$ & 0 \\
\hline & & $\mathrm{O}$ & $8 \mathrm{~g}$ & $0.280(4)$ & $0.280(4)$ & 0 \\
\hline \multirow[t]{9}{*}{1} & $\mathrm{a}=\mathrm{b}=7.436(6)$ & $\mathrm{Fe}$ & $2 a$ & 0 & 0 & 0 \\
\hline & $\mathrm{c}=10.53(1)$ & $\mathrm{Mn}$ & $2 b$ & 0 & 0 & 0.5 \\
\hline & & $\mathrm{Rb} 1$ & $2 \mathrm{c}$ & 0 & 0.5 & 0.25 \\
\hline & & $\mathrm{Rb} 2$ & $2 d$ & 0 & 0.5 & 0.75 \\
\hline & & Ba1 & $2 c$ & 0 & 0.5 & 0.25 \\
\hline & & $\mathrm{Ba} 2$ & $2 d$ & 0 & 0.5 & 0.75 \\
\hline & & $\mathrm{C}$ & $8 \mathrm{~g}$ & $0.205(6)$ & $0.205(6)$ & 0 \\
\hline & & $\mathrm{N}$ & $8 \mathrm{~g}$ & $0.279(9)$ & $0.279(9)$ & 0 \\
\hline & & $\mathrm{O}$ & $8 \mathrm{~g}$ & $0.279(9)$ & $0.279(9)$ & 0 \\
\hline \multirow[t]{9}{*}{0} & $\mathrm{a}=\mathrm{b}=7.439(7)$ & $\mathrm{Fe}$ & $2 a$ & 0 & 0 & 0 \\
\hline & $\mathrm{c}=10.53(1)$ & Mn & $2 b$ & 0 & 0 & 0.5 \\
\hline & & $\mathrm{Rb} 1$ & $2 c$ & 0 & 0.5 & 0.25 \\
\hline & & $\mathrm{Rb} 2$ & $2 d$ & 0 & 0.5 & 0.75 \\
\hline & & Ba1 & $2 \mathrm{c}$ & 0 & 0.5 & 0.25 \\
\hline & & $\mathrm{Ba} 2$ & $2 d$ & 0 & 0.5 & 0.75 \\
\hline & & $\mathrm{C}$ & $8 \mathrm{~g}$ & $0.205(3)$ & $0.205(3)$ & 0 \\
\hline & & $\mathrm{N}$ & $8 \mathrm{~g}$ & $0.281(0)$ & $0.281(0)$ & 0 \\
\hline & & $\mathrm{O}$ & $8 \mathrm{~g}$ & $0.281(0)$ & $0.281(0)$ & 0 \\
\hline
\end{tabular}

vs $T$ curves. As $H$ is increased, the peak at $T_{\mathrm{N}}$ shifts to lower temperatures. Also, there is a clear evidence for the existence of another transition at higher temperatures, which is seen more clearly in the $\mathrm{d} \chi_{\mathrm{ac}} / \mathrm{d} T$ vs $T$ curves [Fig. 7(b)]. This transition, as we know from our field dependent neutron diffraction study, corresponds to a change from an antiferromagnetic into a ferrimagnetic state and the shoulder may be interpreted as $T_{\mathrm{C}}$. Our neutron diffraction results, shown in Figs. 1 and 2, also corroborate the coexistence of antiferromagnetic and ferrimagnetic phases under higher values of $H$. The shift in $T_{\mathrm{N}}$ and evolution of $T_{\mathrm{C}}$ are manifested more clearly in the $\mathrm{d} \chi_{\mathrm{ac}} / \mathrm{d} T$ vs $T$ curves, shown in Fig. 7(b). To show the evolution of the FIM phase more clearly, an enlarged view of the $\mathrm{d} \chi_{\mathrm{ac}} / \mathrm{d} T$ vs $T$ curves is plotted in Fig. 7(c). The values of $T_{\mathrm{N}}$ and $T_{\mathrm{C}}$ are estimated from these plots.

We propose a magnetic phase diagram of the present $\mathrm{Rb}_{0.19} \mathrm{Ba}_{0.3} \mathrm{Mn}_{1.1}\left[\mathrm{Fe}(\mathrm{CN})_{6}\right] \cdot 0.48 \mathrm{H}_{2} \mathrm{O}$ PBA (Fig. 8). We have seen from Fig. 4(c) that at $12 \mathrm{~K}$, the onset of the ferrimagnetic transition occurs between 0 and $1 \mathrm{~T}$. So, a data point has been put in the phase diagram accordingly. We observe that the pure AFM phase exists only over a narrow region of $T$ - $H$ plane. The AFM-FIM transition is very broad, and the two phases coexist for $0 \leq H \leq 4 \mathrm{~T}$. Since, the sample is polycrystalline in nature, the easy direction of magnetic moment is distributed at random in the sample. The effect of magnetic field on each crystallite, therefore varies and leads to a broad transition from AFM to FIM phase. Another important observation in the phase diagram is the presence of a tricritical point at $\sim 12 \mathrm{~K}$ and $0.5 \mathrm{~T}$ where three-phases (antiferromagnetic, ferrimagnetic and paramagnetic) coexist.

As already discussed that the spin configuration of $\mathrm{Rb}_{0.19} \mathrm{Ba}_{0.3} \mathrm{Mn}_{1.1}\left[\mathrm{Fe}(\mathrm{CN})_{6}\right] \cdot 0.48 \mathrm{H}_{2} \mathrm{O}$ under external field is different from the magnetic ground state (see Figs. 6(a) and 6(b)). The mechanism of the AFM-FIM transition can be understood on the basis of the magnetic orbital interactions in the compound.

In a PBA with general formula $A_{x}\left[B(\mathrm{CN})_{6}\right]_{y} \cdot z \mathrm{H}_{2} \mathrm{O}$, the exchange constant $J$ may be expressed as ${ }^{42,46,47}$ 


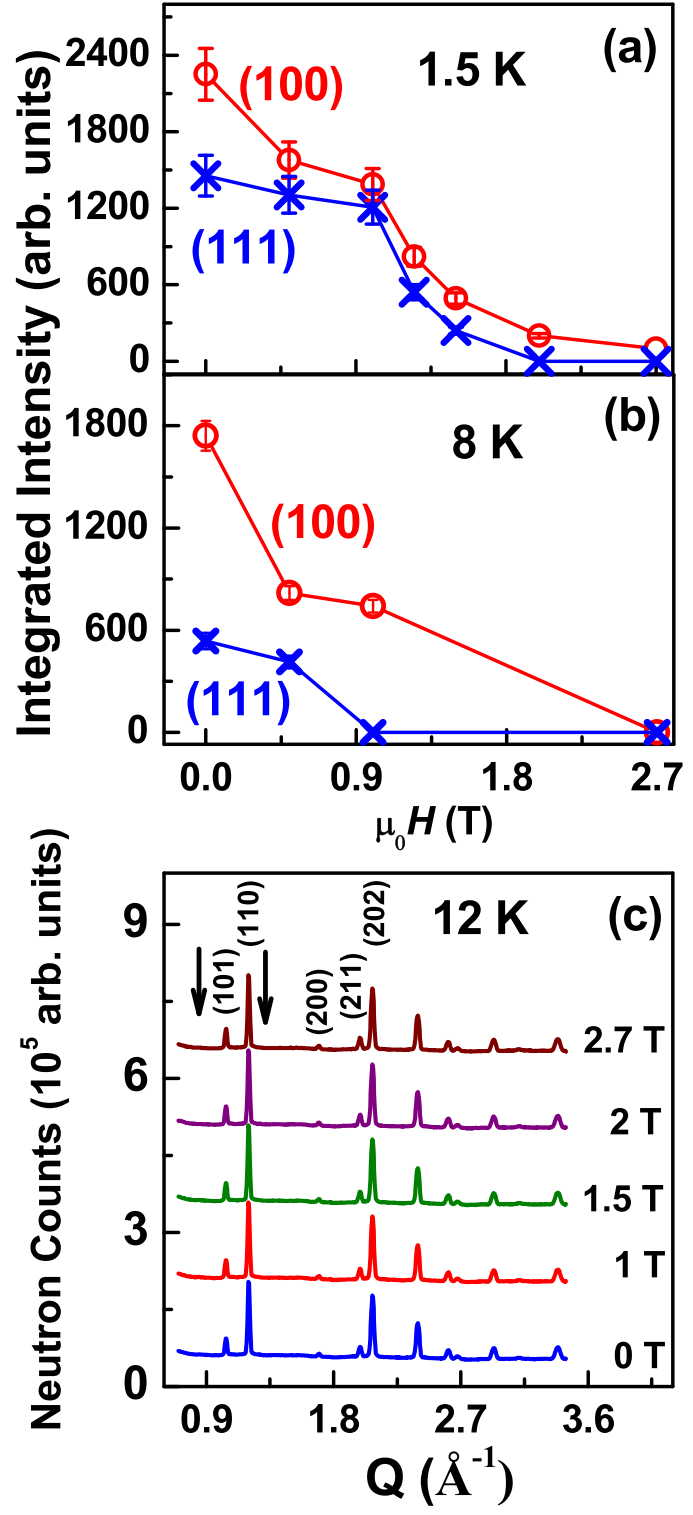

FIG. 3. Variation of intensities with applied magnetic field $(H)$ of antiferromagnetic (100) and (111) Bragg peaks at (a) $1.5 \mathrm{~K}$ and (b) $8 \mathrm{~K}$. (c) Neutron diffraction patterns at $12 \mathrm{~K}$ under different values of $H$. The $(h \mathrm{kl})$ values of the first few fundamental Bragg peaks are given. The arrows mark the positions of the expected antiferromagnetic (100) and (111) Bragg peaks. The curves have been shifted along $y$ axis for clarity.

$$
J=\frac{\sum J_{i j}}{n_{A} n_{B}} .
$$

Here, $J_{i j}$ is the contribution due to the interaction between magnetic orbitals $i$ on $A$ and $j$ on $B$, while $n_{A}$ and $n_{B}$ are the number of unpaired electrons on $A$ and $B$, respectively. $J_{i j}$ may be expressed as a sum of a ferromagnetic term and an antiferromagnetic term

$$
J_{i j}=J_{i j}^{F}+J_{i j}^{A F},
$$

where $J_{i j}{ }^{F}(>0)$ is the exchange constant for a ferromagnetic coupling between $A$ and $B$ which arises due to an orthogonality of their magnetic orbitals. While, $J_{i j}{ }^{A F}(<0)$ is the exchange constant for an antiferromagnetic coupling between $A$ and $B$ which arises due to a non-orthogonality

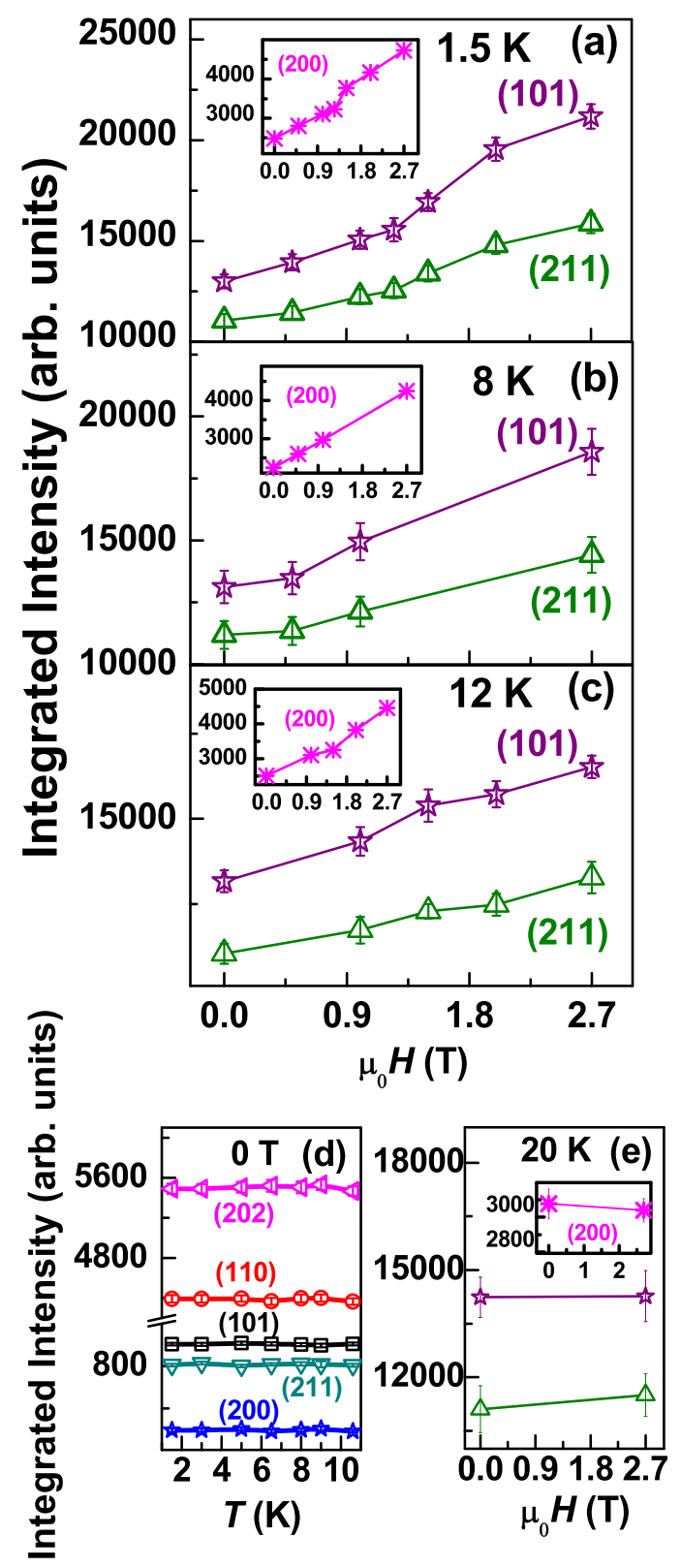

FIG. 4. Variation of intensities with applied magnetic field $(H)$ of fundamental (101), (211) and (200) Bragg peaks at (a) $1.5 \mathrm{~K}$, (b) $8 \mathrm{~K}$ and (c) $12 \mathrm{~K}$. (d) Variation of intensities of fundamental (101), (110), (200), (211) and (202) Bragg peaks with temperature at zero applied magnetic field. (e) Variation of intensities with applied magnetic field $(H)$ of fundamental (101), (211) and (200) Bragg peaks at $20 \mathrm{~K}$.

(overlap) of their magnetic orbitals. ${ }^{48}$ If $J_{i j}{ }^{F}>J_{i j}{ }^{A F}$, the dominant interaction will be ferromagnetic, and if $J_{i j}{ }^{F}<$ $J_{i j}{ }^{A F}$ the dominant interaction will be antiferromagnetic.

Now, we apply the above discussed concept to the studied PBA. The electronic configuration of $\mathrm{Mn}^{2+}$ is $t_{2 g}{ }^{3} e_{g}{ }^{2}$ while that of $\mathrm{Fe}^{3+}$ is $t_{2 g}{ }^{5} e_{g}{ }^{0}$. The $t_{2 g}$ orbitals are $d_{x y}, d_{y z}$ and $d_{z x}$ while $e_{g}$ orbitals are $d_{z 2}$ and $d_{x 2-y 2}$. For $\mathrm{Mn}^{2+}$ ion, unpaired electrons are present in all five $d$ orbitals while for $\mathrm{Fe}^{3+}$ ions, unpaired electrons are present in only $t_{2 g}$ orbitals $\left(d_{x y}, d_{y z}\right.$ and $d_{z x}$ ). Both ferromagnetic (between $e_{g}$ and $t_{2 g}$ via $2 p_{\sigma}$ orbital of $\mathrm{CN}$ ligand) and antiferromagnetic superexchange interactions (between $t_{2 g}$ and $t_{2 g}$ via $2 p_{\pi}$ orbital of $\mathrm{CN}$ ligand) coexist between $\mathrm{Mn}^{2+}$ and $\mathrm{Fe}^{3+}$ ions (see 


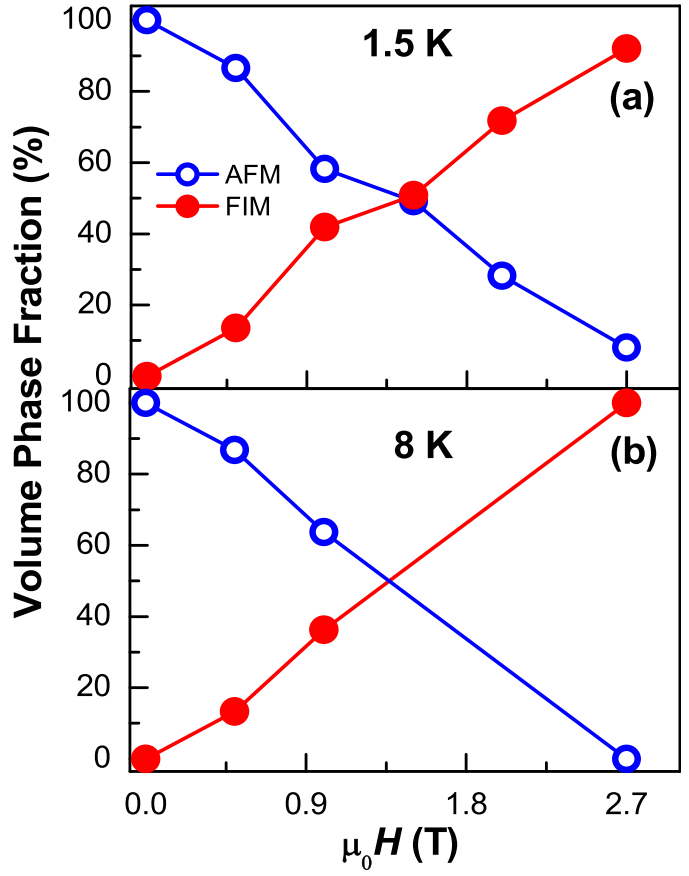

FIG. 5. Variations of the AFM and the FIM phase fractions with applied magnetic field at (a) $1.5 \mathrm{~K}$. (b) $8 \mathrm{~K}$.

Eq. (2)). In the crystallographic $z(c)$ direction, the orientations of the Mn $t_{2 g}$ orbitals and $\mathrm{Fe} t_{2 g}$ orbitals are such that these orbitals are non-orthogonal to $\mathrm{p}_{\pi}$ orbital of $\mathrm{CN}$ ligand (only Mn- $d_{x y}$ magnetic orbital is shown in Fig. 6 for clarity). This leads to an antiferromagnetic interaction between $\mathrm{Mn}^{2+}$ and $\mathrm{Fe}^{3+}$ spins along the crystallographic $z(c)$ axis. It may be noted that magnetic orbital interactions leading to a ferromagnetic exchange also exist in these directions; however, they are dominated by an antiferromagnetic exchange. In the $x y(a b)$ plane, the orientations of the Mn $e_{g}$ orbital and $\mathrm{Fe} t_{2 g}$ orbital are such that these orbitals are orthogonal to $p_{\sigma}$ orbital of $\mathrm{CN}$ ligand giving rise to a ferromagnetic superexchange within this plane. Also, the non-orthogonality of $\mathrm{Mn} t_{2 g}$ and Fe $t_{2 g}$ orbitals to $p_{\pi}$ orbital of CN ligand gives rise to an antiferromagnetic superexchange within $x y(a b)$ plane (as shown in Fig. 6(b)). Both ferromagnetic and antiferromagnetic superexchange interactions (via $\mathrm{CN}$ ligand) coexist between $\mathrm{Mn}^{2+}$ and $\mathrm{Fe}^{3+}$ ions in the $x y(a b)$ plane and compete with each other. In the absence of an external magnetic field, the ferromagnetic superexchange is dominant in $x y$ plane (i.e., $J_{i j}{ }^{F}>J_{i j}{ }^{A F}$ ). Thus the zero field magnetic ground state of the compound is antiferromagnetic (Fig. 6(a). However, in the presence of an external magnetic field, the dominant superexchange interaction in $x y$ plane changes from ferromagnetic to antiferromagnetic (i.e., $J_{i j}{ }^{F}<J_{i j}{ }^{A F}$ ). ${ }^{49}$ The dominance of antiferromagnetic pathways could be possible due to an increased overlap of the $t_{2 g}-t_{2 g}$ magnetic orbitals on applying magnetic field. Consequently, the magnetic state of these compounds under an external magnetic field is ferrimagnetic, as shown in Fig. 6(b). The observed AFM-FIM phase transition could be ascribed to a number of factors, as already reported in the literature. For example, Miyasaka et al. ${ }^{49}$ reported that in the cyanide-ligand bridged compound $\left[\mathrm{NEt}_{4}\right][\mathrm{Mn}(5-\mathrm{Cl} \text {-salen })]_{2}\left[\mathrm{Fe}(\mathrm{CN})_{6}\right]$, small changes in the

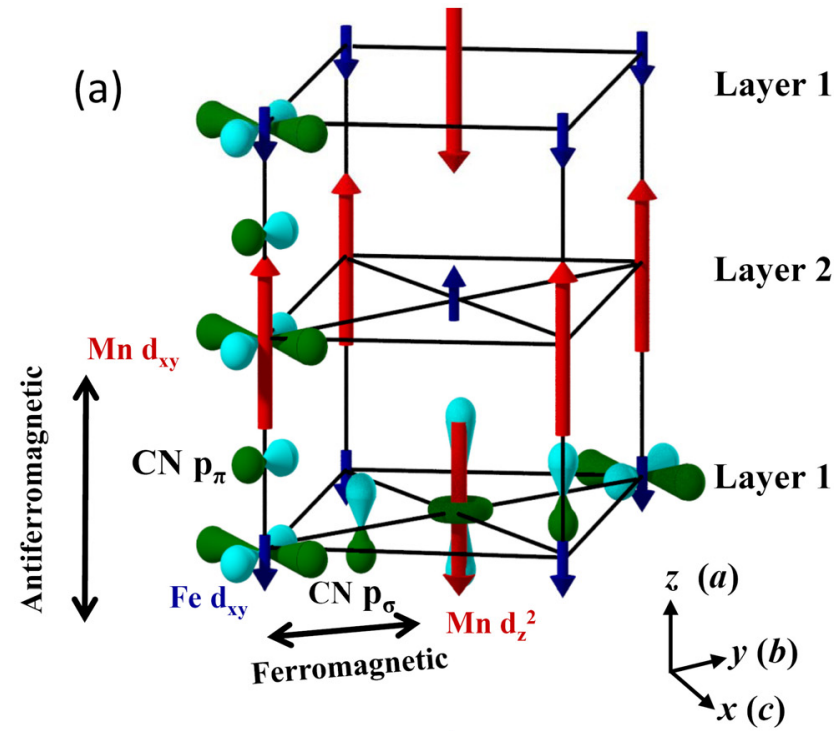

(b)

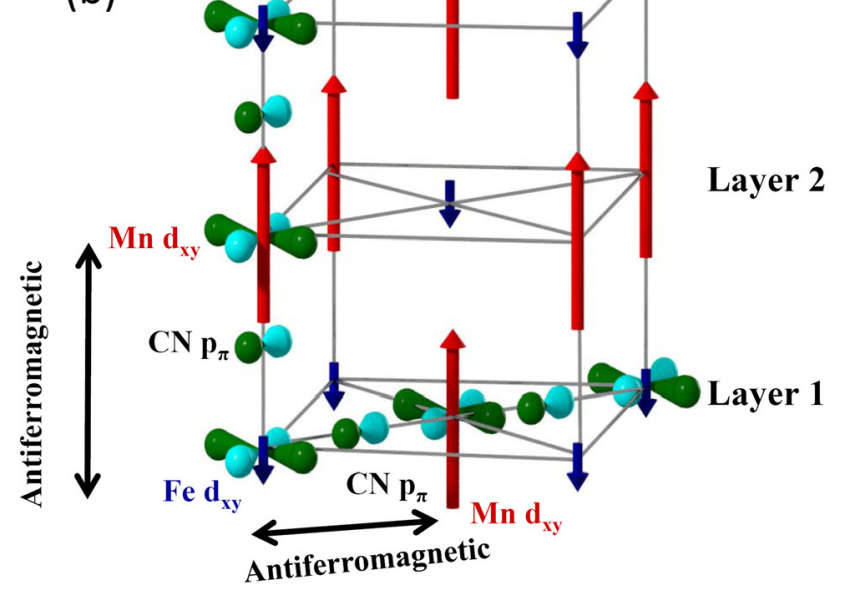

FIG. 6. (a) Magnetic structure under zero magnetic field (ground state) at $1.5 \mathrm{~K}$ (one unit cell is shown). (b) Layered ferrimagnetic structure in the presence of applied magnetic field at $1.5 \mathrm{~K}$ (one unit cell is shown). Large (red) arrows and small (blue) arrows correspond to the ordered $\mathrm{Mn}^{2+}$ and $\mathrm{Fe}^{3+}$ magnetic moments, respectively, along the crystallographic $c$-axis. Layer 1 and 2 denote the layers of magnetic ions in $a b$ plane. Magnetic orbitals are also shown along $z(c)$ axis and in $x y(a b)$ plane to view the superexchange pathways between $\mathrm{Mn}^{2+}$ and $\mathrm{Fe}^{3+}$ ions via $\mathrm{CN}$ ligand.

geometry of the Fe-C-N-Mn unit could lead to an inversion of the magnetic coupling between the two ions. Such type of AFM-FIM phase transition has been also reported by Jiang et $a .^{38}$ in cyanide-ligand bridged compound $\left\{\left[(\mathrm{Tp}) \mathrm{Fe}^{\mathrm{III}}\right.\right.$ $\left.\left.(\mathrm{CN})_{3}\right]_{2} \mathrm{Mn}^{\mathrm{II}}-(\mathrm{DMF})_{2}\right\}_{\mathrm{n}}$.

Now, we compare the field induced behavior of the studied PBA with those of $\mathrm{CoCl}_{2} \cdot 2 \mathrm{H}_{2} \mathrm{O}$ and $\mathrm{FeCl}_{2} \cdot 2 \mathrm{H}_{2} \mathrm{O} .{ }^{30}$ Both these chlorides have an antiferromagnetic structure which undergoes a first-order phase transition to a ferrimagnetic phase under application of magnetic field at low temperatures. ${ }^{30} \mathrm{~A}$ second first-order transition (from the ferrimagnetic phase to a paramagnetie phase) occurs in these chlorides at higher field strengths. ${ }^{30}$ The $\mathrm{Rb}_{0.19} \mathrm{Ba}_{0.3} \mathrm{Mn}_{1.1}$ $\left[\mathrm{Fe}(\mathrm{CN})_{6}\right] \cdot 0.48 \mathrm{H}_{2} \mathrm{O}$ PBA investigated in the present study shows a transition from an AFM to a mixed (AFM+FIM) and then to a FIM phase upon application of magnetic field up to $4 \mathrm{~T}$. A magnetic field greater than $4 \mathrm{~T}$ is required to 


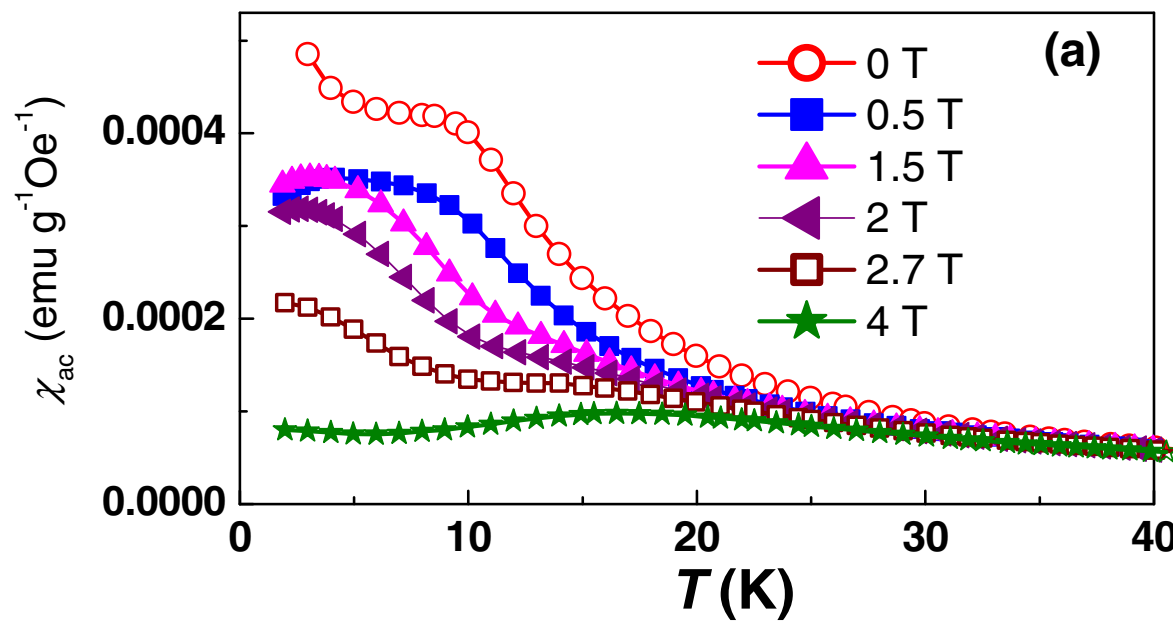

FIG. 7. (a) Variation of real part of ac susceptibility $\left(\chi_{\text {ac }}\right)$ with temperature $(T)$ at different values of applied dc magnetic field. (b) Variation of derivative of $\chi_{\mathrm{ac}}\left(\mathrm{d} \chi_{\mathrm{ac}} / \mathrm{d} T\right)$ with $T$ at different values of applied magnetic field. The curves have been shifted along $y$ axis for clarity. The slanted line depicts the shifting of Néel temperature $\left(T_{\mathrm{N}}\right)$ towards lower temperatures with increasing magnetic field. The encircled region depicts the development of a ferrimagnetic phase in the compound with increasing magnetic field. (c) An enlarged view of $\mathrm{d} \chi_{\mathrm{ac}} / \mathrm{d} T$ vs $T$ curves to show the evolution of ferrimagnetic phase more clearly.
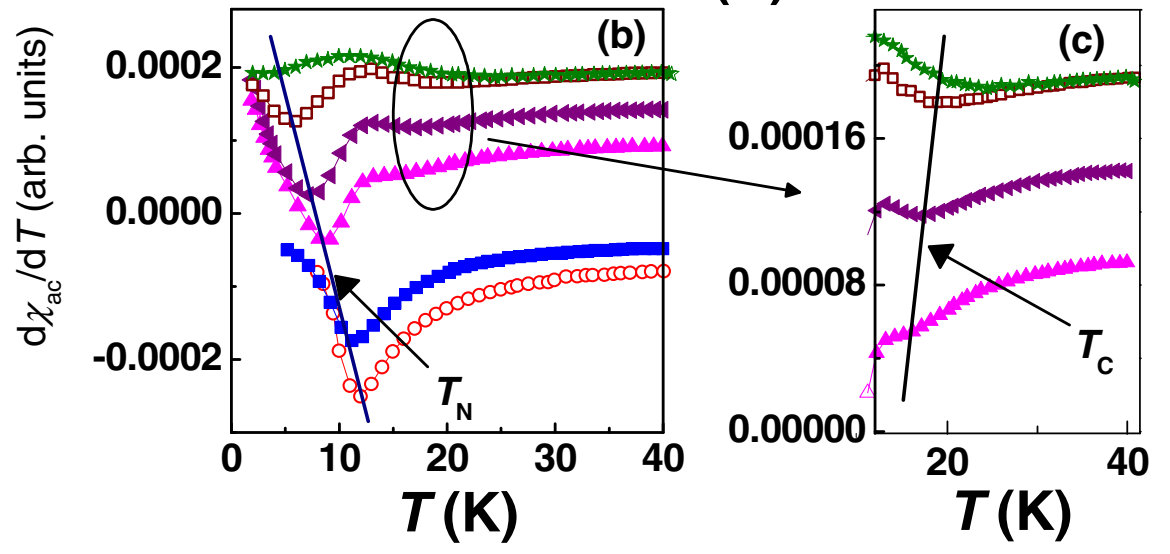

investigate any further magnetic phase transition. In the $\mathrm{CoCl}_{2} \cdot 2 \mathrm{H}_{2} \mathrm{O}$ and $\mathrm{FeCl}_{2} \cdot 2 \mathrm{H}_{2} \mathrm{O}$ compounds, the three phases: antiferromagnetic, ferrimagnetic and paramagnetic meet at a triple point. ${ }^{30}$ For the present system also, a triple point

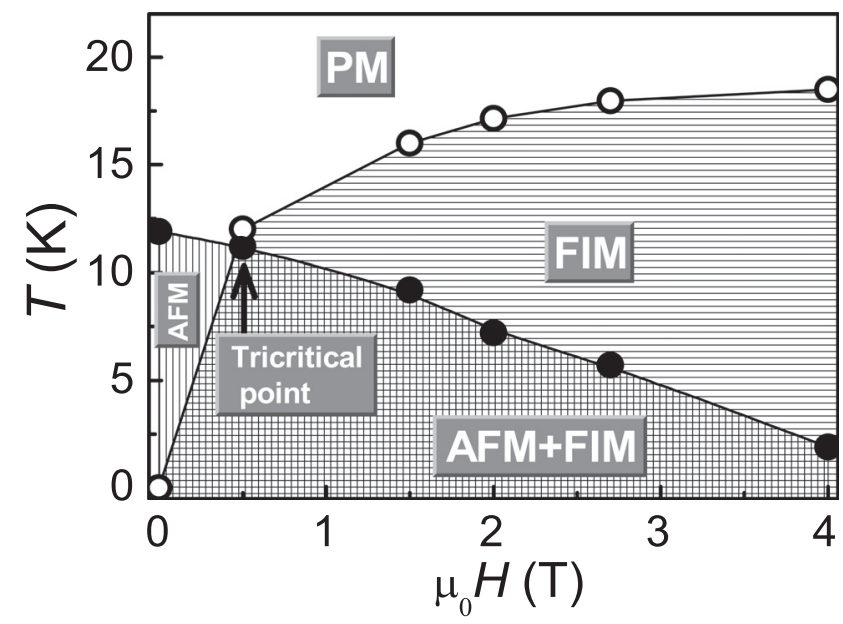

FIG. 8. Magnetic phase diagram of the $\mathrm{Rb}_{0.19} \mathrm{Ba}_{0.3} \mathrm{Mn}_{1.1}\left[\mathrm{Fe}(\mathrm{CN})_{6}\right] \cdot 0.48 \mathrm{H}_{2} \mathrm{O}$ PBA. Vertical and horizontal lines denote the antiferromagnetic (AFM) and ferrimagnetic (FIM) phases, respectively. The region depicting squares denotes the mixed phase (AFM+FIM). Plane white region is the paramagnetic (PM) phase. Solid circles denote the antiferromagnetic Néel temperature $\left(T_{\mathrm{N}}\right)$ and hollow circles denote the ferrimagnetic transition temperature $\left(T_{C}\right)$. The values of $T_{\mathrm{N}}$ have been obtained from dc magnetic field dependent ac susceptibility measurements. The values of $T_{\mathrm{C}}$ for $H \geq 1.5 \mathrm{~T}$ (the last four data points) have been obtained from dc magnetic field dependent ac susceptibility measurements (Fig. 7). The values of $T_{\mathrm{C}}$ for $H<1.5 \mathrm{~T}$ (the first two data points) have been obtained from dc magnetic field dependent neutron diffraction measurements (Fig. 4(c)). exists at the meeting point of the three phases: antiferromagnetic, ferrimagnetic and paramagnetic.

\section{SUMMARY AND CONCLUSIONS}

In summary, we have achieved a microscopic understanding of the magnetic field induced antiferromagnetic to ferrimagnetic phase transition in $\mathrm{Rb}_{0.19} \mathrm{Ba}_{0.3} \mathrm{Mn}_{1.1}$ $\left[\mathrm{Fe}(\mathrm{CN})_{6}\right] \cdot 0.48 \mathrm{H}_{2} \mathrm{O}$ PBA with the help of magnetic field dependent neutron diffraction. Ac susceptibility measurements under dc magnetic field have helped us to determine the variation of antiferromagnetic $\left(T_{\mathrm{N}}\right)$ and ferrimagnetic $\left(T_{\mathrm{C}}\right)$ transition temperatures with $H$. A magnetic phase diagram has been proposed for this system. It is observed that the pure AFM phase exists in only a narrow $T-H$ region. There is a broad region (in the $T-H$ plane) of coexisting AFM and FIM phases. Above $4 \mathrm{~T}$, only FIM phase exists. The AFM-FIM transition is very broad and the two phases coexist for $0 \leq H$ $\leq 4 \mathrm{~T}$. An important finding is the presence of a tricritical point at the meeting point of the three phases: antiferromagnetic, ferrimagnetic and paramagnetic. The ferrimagnetic structure of the compound under external magnetic field consists of antiferromagnetically coupled layers. Within each layer, $\mathrm{Mn}$ and $\mathrm{Fe}$ spins are aligned in antiparallel directions. The observed magnetic phase transition is seen in the light of orbital model of superexchange interaction between magnetic ions $\left(\mathrm{Mn}^{2+}\right.$ and $\left.\mathrm{Fe}^{3+}\right)$ mediated by cyanide ligands.

${ }^{1}$ O. Kahn, Molecular Magnetism (VCH, New York, 1993).

${ }^{2}$ P. Day, Notes Rec. R. Soc. London 56, 95 (2002).

${ }^{3}$ S. J. Blundell and F. L. Pratt, J. Phys.: Condens. Matter. 16, R771 (2004). 
${ }^{4}$ J. S. Miller and A. J. Epstein, MRS Bull. 25, 21 (2000).

${ }^{5}$ S. Ferlay, T. Mallah, R. Ouahes, P. Veillet, and M. Verdaguer, Nature 378, 701 (1995).

${ }^{6}$ O. Hatlevik, W. E. Buschmann, J. Zhang, J. L. Manson, and J. S. Miller, Adv. Mater. 11, 914 (1999)

${ }^{7}$ W. R. Entley and G. Girolami, Science 268, 397 (1995).

${ }^{8}$ S. M. Holmes and S. G. Girolami, J. Am. Chem. Soc. 121, 5593 (1999).

${ }^{9}$ A. Kumar, S. M. Yusuf, and L. Keller, Phys. Rev. B 71, 054414 (2005).

${ }^{10}$ A. Kumar, S. M. Yusuf, L. Keller, J. V. Yakhmi, J. K. Srivastava, and P. L. Paulose, Phys. Rev. B 75, 224419 (2007).

${ }^{11}$ A. Kumar, S. M. Yusuf, L. Keller, and J. V. Yakhmi, Phys. Rev. Lett. 101, 207206 (2008).

${ }^{12}$ A. Widmann, H. Kahlert, I. Petrovic-Prelevic, H. Wulff, J. V. Yakhmi, N. Bagkar, and F. Scholz, Inorg. Chem. 41, 5706 (2002).

${ }^{13}$ H. Miyasaka, H. Takahashi, T. Madanbashi, K. I. Sugiura, R. Clèrac, and H. Nojiri, Inorg. Chem. 44, 5969 (2005).

${ }^{14}$ P. Franz, C. Ambrus, A. Hauser, D. Chernyshov, M. Hostettler, J. Hauser, L. Keller, K. Krämer, H. Stoeckli-Evans, P. Pattison, H.-B. Bürgi, and S. Decurtins, J. Am. Chem. Soc. 126, 16472 (2004).

${ }^{15}$ N. Bagkar, S. Choudhury, K. H. Kim, P. Chowdhury, S. I. Lee, and J. V. Yakhmi, Thin Solid Films 513, 325 (2006).

${ }^{16}$ N. Bagkar, A. Widmann, H. Kahlert, G. Ravikumar, S. M. Yusuf, F. Scholz, and J. V. Yakhmi, Philos. Mag. 85, 3659 (2005).

${ }^{17}$ S. Choudhury, G. K. Dey, and J. V. Yakhmi, J. Cryst. Growth 258, 197 (2003).

${ }^{18}$ W. Dong, L. N. Zhu, H. B. Song, D. Z. Liao, Z. H. Jiang, S. P. Yan, P. Cheng, and S. Gao, Inorg. Chem. 43, 2465 (2004).

${ }^{19}$ A. Kumar and S. M. Yusuf, Physica B 362, 278 (2005).

${ }^{20}$ Z. Lu, X. Wang, Z. Liu, F. Liao, S. Gao, R. Xiong, H. Ma, D. Zhang, and D. Zhu, Inorg. Chem. 45, 999 (2006).

${ }^{21}$ S. Ohkoshi, K.-I. Arai, Y. Sato, and K. Hashimoto, Nature Mater. 3, 857 (2004).

${ }^{22}$ O. Sato, Y. Einaga, T. Iyoda, A. Fujishima, and K. Hashimoto, J. Electrochem. Soc. 144, L11 (1997).

${ }^{23}$ O. Sato, T. Iyoda, A. Fujishima, and K. Hashimoto, Science 272, 704 (1996).

${ }^{24}$ J. V. Yakhmi, Physica B 321, 204 (2002).

${ }^{25}$ S. M. Yusuf, N. Thakur, A. Kumar, and J. V. Yakhmi, J. Appl. Phys. 107, 053902 (2010).

${ }^{26}$ J. V. Yakhmi, Bull. Mater. Sci. (India) 32, 217 (2009).
${ }^{27}$ N. Thakur, S. M. Yusuf, and J. V. Yakhmi, Phys. Chem. Chem. Phys 12, 12208 (2010).

${ }^{28}$ E. Coronado, M. C. Giménez-López, G. Levchenko, F. M. Romero, V. García-Baonza, A. Milner, and M. Paz-Pasternak, J. Am. Chem. Soc. 127, 4580 (2005).

${ }^{29}$ S. Ohkoshi, H. Tokoro, and K. Hashimoto, Coord. Chem. Rev. 249, 1830 (2005).

${ }^{30}$ E. Stryjewski and N. Giordano, Adv. Phys. 26, 487 (1977).

${ }^{31}$ I. S. Jacobs and P. E. Lawrence, Phys. Rev. 164, 866 (1967)

${ }^{32}$ M. K. Wilkinson, J. W. Cable, E. O. Wollan, and W. C. Koehler, Phys. Rev. 113, 497 (1959).

${ }^{33}$ R. J. Birgeneau, G. Shirane, M. Blume, and W. C. Koehler, Phys. Rev. Lett. 33, 1098 (1974).

${ }^{34}$ L. J. DeJongh and A. R. Miedema, Adv. Phys. 23, 1 (1974).

${ }^{35}$ D. E. Cox, G. Shirane, B. C. Frazer, and A. Narath, J. Appl. Phys. 37, 1126 (1966).

${ }^{36}$ R. D. Spence and A. C. Botterman, Phys. Rev. B 9, 2993 (1974).

${ }^{37}$ A. Narath, J. Phys. Soc. Jpn. 19, 2244 (1964).

${ }^{38}$ L. Jiang, X. Feng, T. Lu, and S. Gao, Inorg. Chem. 45, 5018 (2006).

${ }^{39}$ E. Colacio, J. M. Domínguez-Vera, M. Ghazi, R. Kivekäs, F. Lloret, J. M. Morenoa, and H. Stoeckli-Evans, Chem. Commun. 11, 987 (1999).

${ }^{40}$ H. Miyasaka, H. Okawa, A. Miyazaki, and T. Enoki, Inorg. Chem. 37, 4878 (1998).

${ }^{41}$ R. Herchel, J. Tuček, Z. Trávníček, D. Petridis, and R. Zbořil, Inorg. Chem. 50, 9153 (2011).

${ }^{42}$ A. Bhattacharjee, S. Saha, S. Koner, V. Ksenofontov, S. Reiman, and P. Gütlich, J. Magn. Magn. Mater. 302, 173 (2006).

${ }^{43}$ M. K. Saha, M. C. Morón, F. Palacio, and I. Bernal, Inorg. Chem. 44, 1354 (2005)

${ }^{44}$ N. Thakur, S. M. Yusuf, P. L. Paulose, and L. Keller, J. Appl. Phys. 111, 063908 (2012).

${ }^{45}$ J. Rodriguez-Carvajal, FULLPROF, November 2007, http://www.ill.eu/sites/ fullprof/.

${ }^{46} \mathrm{P}$. W. Anderson, Magnetism (Academic, NY, 1963), Vol. 1, p. 25.

${ }^{47} \mathrm{M}$. Verdaguer and G. Girolami, in Magnetism: Molecules to Materials V, edited by J. S. Miller and M. Drillon (Wiley-VCH, 2004).

${ }^{48}$ O. Kahn, Magneto-Structural Correlations in Exchanged Coupled Systems (Kluwer Academic, Dordrecht, 1985).

${ }^{49}$ H. Miyasaka, N. Matsumoto, N. Re, E. Gallo, and C. Floriani, Inorg. Chem. 36, 670 (1997). 